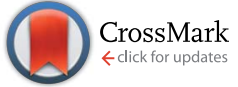

Cite this: RSC Adv., 2017, 7, 3521

Received 14th October 2016

Accepted 11th December 2016

DOI: $10.1039 / \mathrm{c} 6 \mathrm{ra} 25256 \mathrm{~g}$

www.rsc.org/advances

\section{Mineralization of a superficially porous microsphere scaffold via plasma modification}

\author{
Jie Hou, ${ }^{\text {abc }}$ Fen Zhang, ${ }^{\text {abc }}$ Delin Cheng, ${ }^{* d}$ Xuetao Shi ${ }^{\text {abc }}$ and Xiaodong Cao*abc
}

Mineralization on bone repair devices is beneficial for osteoconductivity and thus osteogenesis. Surface chemistry modification by plasma treatment can significantly improve mineralization. In this study, superficially porous poly(lactide-co-glycolide)/hydroxyapatite (PLGA/HA) microsphere scaffolds (PH) were processed by oxygen and ammonia plasma followed by incubation in simulated body fluids (SBF) for mineralization. The effect of plasma treatment and incubation time on the features of the mineralization layer was investigated. The results showed that novel porous mineralization layers were obtained on the unmodified $\mathrm{PH}$ (As-grown), oxygen pretreated $\mathrm{PH}(\mathrm{OPH})$ and ammonia pretreated $\mathrm{PH}$ (NPH). The mechanical property and superficially porous structures of scaffolds changed little after plasma treatment. However, OPH produced the most significant apatite layer with an increasing roughness with mineralization time. In contrast, As-grown just had a smooth mineralization layer. Moreover, the mineralization layer on As-grown was easy to peel off compared with those on $\mathrm{OPH}$ and $\mathrm{NPH}$, demonstrating that plasma treatment could enhance the bonding force between apatite and the substrate surface. The surface chemical analysis proved that the difference in outcome of mineralization was because plasma treatment introduced additional polar domains onto the scaffolds. This work provided a promising mineralization material system for bone repair application.

\section{Introduction}

Superficially porous PLGA/inorganic substance microspheres can be used as microsphere scaffolds in bone tissue engineering aiming to replace natural damaged bones because they have been proven to have good biocompatibility, suitable biodegradation and good-capsulation of anti-inflammatory drugs or growth factors. ${ }^{1}$ Besides, they can be designed in any shape by using a bottom-up way to satisfy patients' demand. Surface morphology with holes has its own advantage of affecting cell behaviours. Tailoring the dimension or topography of surface holes can regulate deposition of proteins, tissue migration and distinguish different kinds of cells. ${ }^{2-4}$ Superficially porous microspheres had the capacity of modulating cell proliferation and differentiation as proved in our previous work. ${ }^{5}$ However, the lack of osteoconductivity of PLGA surface is an obstacle for maximum function in bone repair. Introducing bone-like mineral coatings like hydroxyapatite or other calcium

${ }^{a}$ School of Materials Science and Engineering, South China University of Technology, Guangzhou 510641, China. E-mail: caoxd@scut.edu.cn

${ }^{b}$ National Engineering Research Centre for Tissue Restoration and Reconstruction, Guangzhou 510006, China

${ }^{c}$ Guangdong Province Key Laboratory of Biomedical Engineering, South China University of Technology, Guangzhou 510006, China

${ }^{d}$ Centre for Human Tissue and Organ Degeneration, Shenzhen Institutes of Advanced Technology, Chinese Academy of Sciences, Shenzhen 518055, China. E-mail: $d l$. cheng@siat.ac.cn phosphate onto the material surface can improve and accelerate their integration with bone tissue. ${ }^{6}$ In general, there are mainly two methods to coat hydroxyapatite, namely plasma spraying and mineralization in $\mathrm{SBF}^{7}$ The commercially used plasma spraying will produce high temperature condition and thus destroy the surface fine structure like pores. ${ }^{8} \mathrm{SBF}$ can to some extent, mimic the ion compositions of natural fluid around bone. ${ }^{9}$ Mineralization in SBF has been frequently applied to coat biomimetic hydroxyapatite layer on a variety of biomaterials. $^{\mathbf{1 0}}$

Mineralization in SBF is a complex process with many factors affecting initial nucleation. ${ }^{\mathbf{1 1}}$ Among those factors, surface chemistry of the substrate plays an important role in calcium phosphate nucleation. Liu et al. group found that apatite could deposit on the self-assembled monolayers with $-\mathrm{PO}_{4} \mathrm{H}_{2}$ and $-\mathrm{COOH}$ groups, while not on those with $-\mathrm{CH}=\mathrm{CH}_{2}$ and $-\mathrm{OH}{ }^{12}$ And anionic groups by alkali hydrolysis on the surface of material could effectively accelerate the mineral nucleation in the $\mathrm{SBF}{ }^{\mathbf{1 3}}$

Several methods have been developed to modify the surface chemical properties. ${ }^{14}$ Low-temperature plasma process proves to be a mild and time saving technique to change surface chemistry without destroying surface structures. ${ }^{15}$ It can bring in diverse functional groups and lengthen modification depth in the scaffold. ${ }^{16} \mathrm{Qu}$ et al. increased the content of $\mathrm{C}=\mathrm{O}-\mathrm{O}$ and $\mathrm{C}-\mathrm{O}$ on the PLGA surface using $\mathrm{O}_{2}$ plasma-etching process. ${ }^{17} \mathrm{He}$ et al. applied $\mathrm{NH}_{3}$ plasma to treat PLGA and grafted nitrogen 
functional groups like $\mathrm{N}-\mathrm{H}$ and $\mathrm{C}-\mathrm{N}^{+} .{ }^{18}$ Therefore, it's a good choice to cover and modulate the calcium phosphate coating on the surface using plasma process, especially on superficially porous microsphere scaffolds.

Given the situation mentioned above, in this study, PLGA/HA microspheres with abundant micro-pores were used as raw substrates. The substrates were translated into scaffolds through low-temperature fusion technique. Then they were treated with oxygen or ammonia plasma followed by incubation in SBF for predetermined periods. The outcome of plasma process and the feature of mineralized apatite on the modified substrates were evaluated.

\section{Experimental}

\subsection{Materials}

PLGA (L/G $=85 / 15, M_{\mathrm{w}}=100 \mathrm{kDa}$, ester terminated, inherent viscosity $=0.71 \mathrm{dl} \mathrm{g}^{-1}$ ) was purchased from Daigang Biomaterials (Jinan, China). Poly(vinyl alcohol) (PVA, 87.0-89.0\% hydrolyzed, $\left.M_{\mathrm{w}}=44.05 \mathrm{kDa}\right)$ and $\mathrm{D}-(+)$-gluconic acid $\delta$-lactone (GDL) were obtained from Aladdin Industrial Co. (Shanghai, China). Dichloromethane (DCM) and some inorganic salts which were necessary to prepare SBF bought from Sinopharm Chemical Reagent Co. Ltd. (Shanghai, China).

\subsection{Fabrication of PLGA/HA microsphere scaffold}

PLGA/HA microspheres were prepared by using a single emulsion evaporation method in our previous work. ${ }^{19}$ In brief, $500 \mathrm{mg}$ of PLGA was dissolved in DCM $(10 \%, \mathrm{w} / \mathrm{v})$ as oil phase, obtaining the slurry by addition of $150 \mathrm{mg}$ of HA particles $(2.1$ $\mu \mathrm{m})$. Subsequently, the slurry was injected into $250 \mathrm{ml}$ of $0.6 \%$ (w/v) PVA aqueous solution with $500 \mathrm{mg}$ of GDL and stirred. Finally, the microspheres were washed and collected.

PLGA/HA microsphere scaffolds were fabricated by a lowtemperature fusion technique. ${ }^{20}$ In brief, wet PLGA/HA microspheres were poured into Teflon cylindrical molds $(6 \mathrm{~mm} \times 5$ $\mathrm{mm}$ ) and then put them in the incubator at $37^{\circ} \mathrm{C}$ for $48 \mathrm{~h}$.

\subsection{Plasma process}

Plasma treatment was utilized to change the chemical condition of microsphere unit surfaces. PLGA/HA microsphere scaffolds were put into plasma processor (MiniFlecto, plasmatechnology, German) and vacuumized to 0.07 mbar after sealing. The pressure was maintained for $120 \mathrm{~s}$ at $0.3 \mathrm{mbar}$ after bubbling $\mathrm{O}_{2}$ or $\mathrm{NH}_{3}$ with an air flow rate of $8 \mathrm{sccm}$. Then the gas was in radio frequency glow discharge at low temperature. The plasma treatment was carried out for $5 \mathrm{~min}$ at $80 \mathrm{~W}$ and the air flow rate was 6-7 sccm.

\subsection{Mineralization}

Mineralization was carried out in a $5 \times$ SBF. The SBF was prepared according to Barrere's recipe. ${ }^{21}$ Then microsphere scaffolds were soaked in the SBF system and placed in the shaker $\left(37^{\circ} \mathrm{C}, 90 \mathrm{rpm}\right)$ for appropriated time (0.5, 2 and $\left.7 \mathrm{~h}\right)$. The mineralized microsphere scaffolds were rinsed with deionized water several times and lyophilized.

\subsection{Characterization}

The surfaces of the microsphere scaffolds before and after mineralization were characterized by scanning electron microscope (SEM, Merlin, Zeiss, Germany). The samples were sputtercoated with platinum by using coater (Quorum, England). The $\mathrm{Ca} / \mathrm{P}$ ratios of mineralization layers on the three scaffolds were detected by using energy dispersive X-ray detector (EDX, 30XLFEG, Philips, the Netherlands). The compressive strength of microsphere scaffolds was evaluated by using a mechanical testing machine (Instron 5967, INSTRON, America) with a crosshead speed of $0.1 \mathrm{~mm} \mathrm{~s}^{-1}$ at ambient temperature and humidity $(n=4)$. The pore diameters on the microsphere surfaces of the three scaffolds were calculated by Image J software $(n=4)$.

The inorganic constituent of the mineralized microsphere scaffolds at the mineralization time of $7 \mathrm{~h}$ was detected by using X-ray diffractometer (XRD, Empyrean, PANalytical, Holland) with thin film diffraction plug-in and a monochromatic $\mathrm{Cu} \mathrm{K \alpha}$ source. $200 \mathrm{mg}$ of mineralized microspheres was fabricated into cylindrical slice with diameter of $20 \mathrm{~mm}$. XRD patterns were collected over the $2 \theta$ range of $10-80^{\circ}$ at a step size of $0.013^{\circ} \mathrm{s}^{-1}$.

Surface roughness of the samples was examined by atomic force microscopy (AFM, Asylum Research, America) with using a silicon cantilever in tipping mode while maintaining a constant force of $40 \mathrm{~N} \mathrm{~m}^{-1}$ between the tip and the sample. The root mean square (RMS) values were determined by the scan size of $5 \mu \mathrm{m} \times 5 \mu \mathrm{m}$ of the sample $(n=3)$.

Surface chemical compositions of the microsphere scaffolds were investigated by using X-ray photoelectron spectroscopy (XPS, ESCALAB 250, ThermoFisher SCIENTIFIC, America) with a monochromatic Al $\mathrm{K} \alpha$ source. Shirley base line subtraction and Gaussian components were used for core lines. Spectral decomposition and concentrations of various C1s/N1s were calculated from the relative $\mathrm{C} 1 \mathrm{~s} / \mathrm{N} 1 \mathrm{~s}$ peak area.

The zeta potential of the microsphere scaffolds was measured by a solid surface zeta potentiometer (SurPASS, Anton Paar, Austria) with Cylindrical Cell plug-in. The evaluation mode was Fairbrother-Mastin.

\subsection{Statistics analysis}

All data of quantitative experimental results were expressed as mean \pm standard deviations. ANOVA followed by $t$-test was used to compare results of three groups. Statistical significance was defined as $p<0.05$.

\section{Results}

\subsection{The morphologies and compressive strength of As- grown, OPH and NPH}

The SEM images of the three microsphere scaffolds were shown in Fig. 1. As shown in Fig. 1a and b, PLGA/HA microspheres were fused into a scaffold by using the low-temperature fusion method, which was beneficial for the maintenance of the surface topography and stability of microspheres. The PLGA/HA microspheres were screened from $250 \mu \mathrm{m}$ to $450 \mu \mathrm{m}$, and the surface pore size was almost all below $5 \mu \mathrm{m}$. There were multi- 

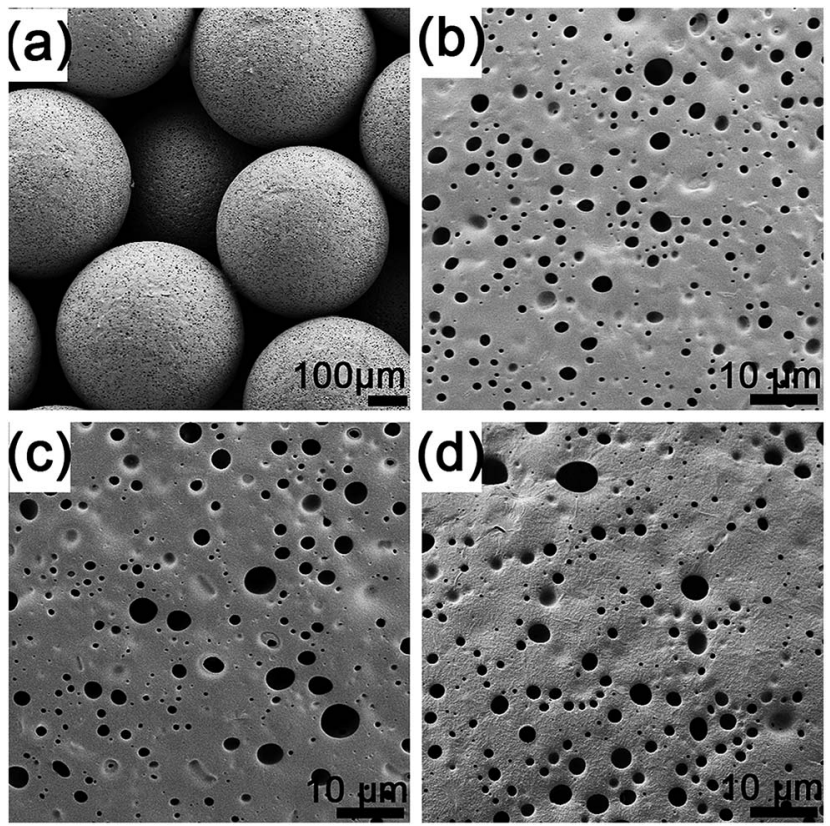

Fig. 1 The SEM images of PLGA/HA microsphere scaffolds: (a) the stacking microspheres of PLGA/HA microsphere scaffold; (b) the surface morphology of microsphere unit; (c) the surface morphology of OPN; (d) the surface morphology of $\mathrm{NPH}$.

level pores, namely small pores on microsphere unit surface and large pores between microspheres of the scaffold. And surface HA was all eliminated by acidic GDL and only PLGA was left behind according to our previous work. ${ }^{22}$ Moreover, the surface pores of PLGA/HA scaffold were well retained after $\mathrm{O}_{2}$ or $\mathrm{NH}_{3}$ plasma treatment. In addition, the pore diameters of $\mathrm{OPH}(1.81 \pm 0.22$ $\mu \mathrm{m})$ and NPH $(1.84 \pm 0.17 \mu \mathrm{m})$ were hardly changed compared with that of As-grown $(1.84 \pm 0.18 \mu \mathrm{m})$. The compressive strength of both $\mathrm{OPH}(5.35 \pm 1.21 \mathrm{MPa})$ and $\mathrm{NPH}(5.45 \pm 0.47 \mathrm{MPa})$ was comparable to that of As-grown $(5.55 \pm 0.95 \mathrm{MPa})$. These were all possibly because low-temperature plasma treatment was mild and confined to the top tens of nanometers without affecting the initial bulk property. The superficially porous substrate provided a novel base for subsequent mineralization.

\subsection{Mineralization}

The morphologies of mineralized microsphere scaffolds with different mineralization time were illustrated in Fig. 2. As for $0.5 \mathrm{~h}$, nearly none apatite deposited on the As-grown surface. However, OPH was decorated with many mineralized clusters while NPH with several small apatite clusters. This indicated that OPH might had the best capability of mineralization while As-grown the least. With mineralization time continuing to $2 \mathrm{~h}$, mineralized apatite layers formed on all the three samples, with open pores distributing all over the surface. Specifically, Asgrown had a smooth mineralization layer and $\mathrm{OPH}$ had a rough one with mineralized clusters. This difference was also due to the difference in capability of mineralization. Moreover, the apatite layer on As-grown showed the sign of peeling (arrow in Fig. 2b), while those on $\mathrm{OPH}$ and NPH still remained intact.
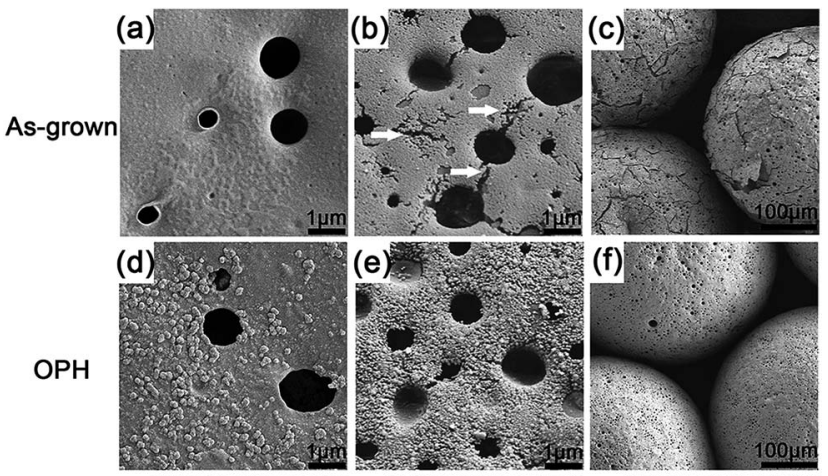

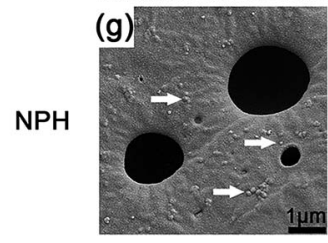

$0.5 \mathrm{~h}$

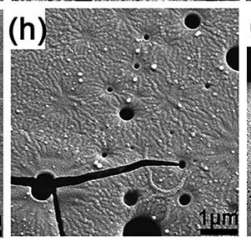

$2 \mathrm{~h}$

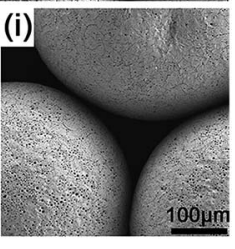

$7 \mathrm{~h}$
Fig. 2 The morphologies of the three microsphere scaffolds after mineralization.

This phenomenon implied that the bonding force between the apatite layer and the substrate was stronger on $\mathrm{OPH}$ and $\mathrm{NPH}$ than on As-grown. When mineralization time was up to $7 \mathrm{~h}$, the peeling trend of apatite layer on As-grown turned obvious. In contrast, OPH and NPH still had intact mineralized surfaces. All these results demonstrated that plasma treatment not only improved the mineralization of microsphere scaffold, but also enhanced the bonding force of apatite layer onto the scaffold.

The composition of mineralization was analysed by XRD in Fig. 3a. The spectra of the three mineralized scaffolds were consistent with that of the raw hydroxyapatite, which demonstrated that mineralization layer was mainly made up of HA. In addition, peak widths at half height of five higher peaks ((002), (211), (112), (300) and (202)) of mineralized scaffolds were broad and two of higher peaks ((211) and (112)) were fused into one. This meant that the crystallization of mineralization layers was low. The $\mathrm{Ca} / \mathrm{P}$ ratios of mineralization layer by $\mathrm{EDX}$ were further detected. As seen in Fig. 3a inset, after 7 hours of immersion the $\mathrm{Ca} / \mathrm{P}$ ratios of the three samples varied between 1.5-1.6, which were lower than the $\mathrm{Ca} / \mathrm{P}$ ratio of $\mathrm{HA}$. This indicated that there was possibly calcium deficiency HA in the surface mineralization layer.

The apatite roughness on the microsphere surface except pores of mineralized scaffolds was also evaluated (Fig. 3b). The surface roughness of the three samples didn't have a significant difference at $0 \mathrm{~h}$. The apatite roughness of $\mathrm{OPH}$ and NPH increased with mineralization time, while As-grown slightly changed. This corresponded to the result of Fig. 2. Due to the weak capability of mineralization, As-grown had a smooth apatite layer with steady roughness. OPH and NPH had better outcome of mineralization, causing the increase of roughness with time.

\subsection{Surface chemistry analysis}

It's noteworthy that negatively charged surface had a significant influence on the apatite mineralization. ${ }^{23}$ The surface chemical 

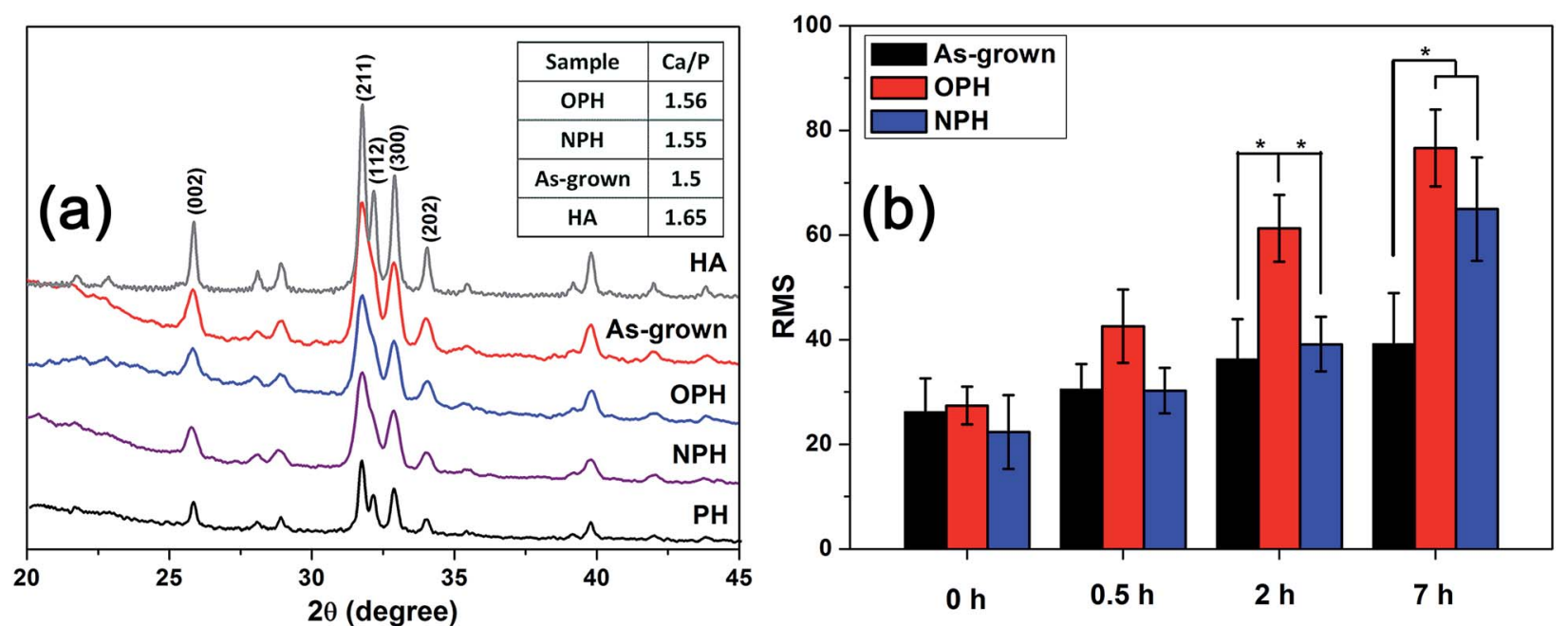

Fig. 3 XRD patterns (a) and apatite roughness (b) of the three microsphere scaffolds after mineralization. The inset in (a) was the Ca/P ratio of mineralization layer on scaffolds at mineralization time of $7 \mathrm{~h}$. * indicated the significant difference when $p<0.05$.

condition of microsphere scaffold before and after plasma processes was determined by XPS. As shown in Table $1, \mathrm{O}_{2}$ plasma treatment led to an increase of oxygen element. And the nitrogen and oxygen element increased after $\mathrm{NH}_{3}$ plasma process. It's demonstrated that plasma processes brought in polar domains $(\mathrm{O} / \mathrm{N})$ replacing hydrogen or carbon to form polar radicals as reported by Koch et al. ${ }^{24}$

To further analyse the change of radical composition, the C1s and N1s peaks were resolved according to valence-bond theory. As shown in Fig. 4 and Table 1, C-H or C-C, C-O or $\mathrm{C}-\mathrm{N}$ and $\mathrm{C}=\mathrm{O}$ peaks were attributed to carbons with binding energies of 284.3, 286 and $288.2 \mathrm{eV}$, respectively ${ }^{25}$ The fractions of $\mathrm{C}-\mathrm{O}$ at $286.2 \mathrm{eV}$ increased on $\mathrm{OPH}$, since some $\mathrm{C}-\mathrm{H} / \mathrm{C}-\mathrm{C}$ bonds were oxidized after oxygen plasma treatment. ${ }^{26}$ Interestingly, the fraction of $\mathrm{C}=\mathrm{O}$ increased sharply on $\mathrm{NPH}$. And there were not only $\mathrm{C}-\mathrm{N}$ and $\mathrm{C}-\mathrm{NH}_{2}$ groups, but also oxidized nitrogen species at $401.6 \mathrm{eV}$ in N1s (Fig. $4 \mathrm{~d}$ ). This was also reported by Torrengo et al. who found that nitrogen functional groups were always accompanied by the presence of oxygen. ${ }^{27}$

Plasma treatment could generate polar domains that had a significant effect on zeta potential of surface. ${ }^{28}$ Altankov et al. fabricated self-assembled monolayers of diverse functional groups and found that the magnitude of negative potential increased following the order of hydroxyl, amino and carboxyl groups. ${ }^{29}$ In this study, the zeta potential of all samples was presented in Fig. 5. As-Grown surface had a slightly linear

Table 1 The element and functional group compositions of the three microsphere scaffold surfaces (\%)

\section{C1s}

\begin{tabular}{llllll} 
Sample & C-C, C-H & C-O, C-N & C=O & O1s & N1s \\
\hline As-Grown & 45.8 & 29.8 & 24.4 & 36.2 & 1.2 \\
OPH & 35.9 & 33.8 & 30.3 & 40.4 & 0 \\
NPH & 31.0 & 31.8 & 37.2 & 39.9 & 4.0
\end{tabular}

decrease of zeta potential with increasing $\mathrm{pH}$. In contrast, zeta potential of plasma pretreated surfaces was lower because of the generated carboxyl groups. Since amino groups were presented on NPH, protonation of amine group resulted in a middle zeta potential. $^{30}$

\section{Discussion}

Plasma treatment can be used to change material surface to benefit HA deposition. Here three microsphere scaffolds with different mineralization layer morphologies were fabricated by using plasma treatment followed by immersion in the SBF. During mineralization in vitro, calcium ions are adsorbed onto the substrate surface, which aggregate near the surface to be the initial nucleation sites. And then phosphate ions are appealed by the positively charged trigger of calcium ions. ${ }^{31}$ So far, many researchers have paid attention to the effect of surface functional groups with different electrical charges on HA precipitation. Most reports indicated that negatively charged functional groups were more effective than positive and neutral groups in inducing heterogeneous nucleation of HA owing to electrostatic attraction and hydrogen bond. ${ }^{32}$ However, there were different points in some cases. Tavafoghi et al. discovered that the positively charged arginine induced faster and more HA precipitation than the negatively charged glutamic. ${ }^{33}$ In fact, for protein or amino acid, not only electrostatic attraction but also other factors like steric-hindrance effect and coupling effect affected the mineralization. ${ }^{34}$ In this paper, there were mainly three simply grafted groups, namely carboxyl, hydroxyl and amino groups. Carboxyl groups with negative charge could accelerate the nucleation of apatite, while amino groups were blocks in inducing apatite formation according to opposite attract theory. ${ }^{35}$ The deprotonation of hydroxyl groups was very weak, which hardly affected the apatite formation. ${ }^{36}$

Classical nucleation theory is the principal explanation of crystallisation in mineralization. The classical view considers the formation of nuclei in supersaturated solution is governed 

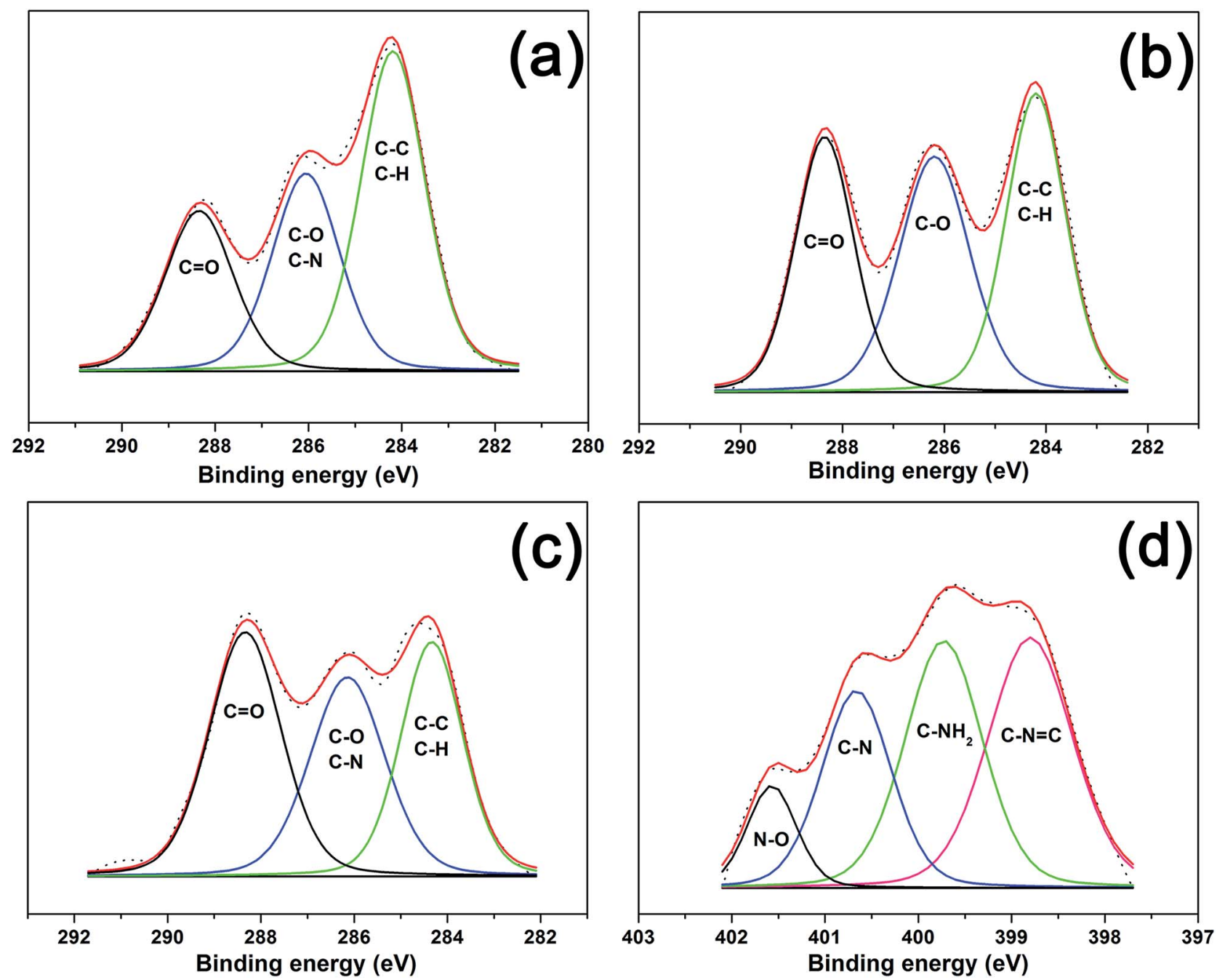

Fig. 4 Normalized XPS core level spectra of the samples: (a) the C1s peaks of As-grown surface; (b) the C1s peaks of OPH surface; (c) the C1s peaks of NPH surface; (d) the N1s peaks of NPH surface.

by the balance between the bulk and surface energy of the new phase. ${ }^{37}$ This notion is divided into homogeneous nucleation and heterogeneous nucleation. However, it was incompatible with several experimental observations in recent years. ${ }^{38} \mathrm{~A}$ prenucleation cluster pathway as a truly non-classical concept of nucleation was put forward and investigated. Posner's model and Habraken's model were carried out, while little was known about the structure and role prenucleation cluster. ${ }^{39,40}$ Combining the previous reports about mineralization on PLGA with data in this study, the effect of plasma process on PLGA surface mineralization was considered as heterogeneous nucleation. ${ }^{41}$

Therefore, the probable mineralization mechanism in this study was proposed as illustrated in Fig. 6. Firstly, As-grown had the least amount of carboxyl groups. The accumulation of calcium ions was too little to appeal phosphate ions for apatite formation on the surface. On the contrary, both OPH and NPH could accelerate apatite formation. And apatite nucleation was greatest on OPH. However, apatite formation suffered obstacles on NPH because amino groups were distributed among

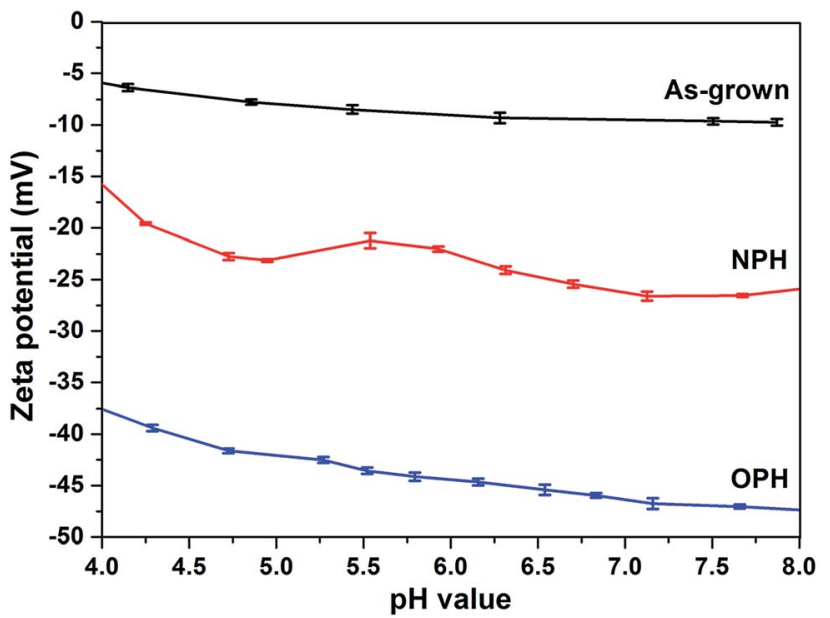

Fig. 5 Surface zeta potential of the three microsphere scaffolds. 

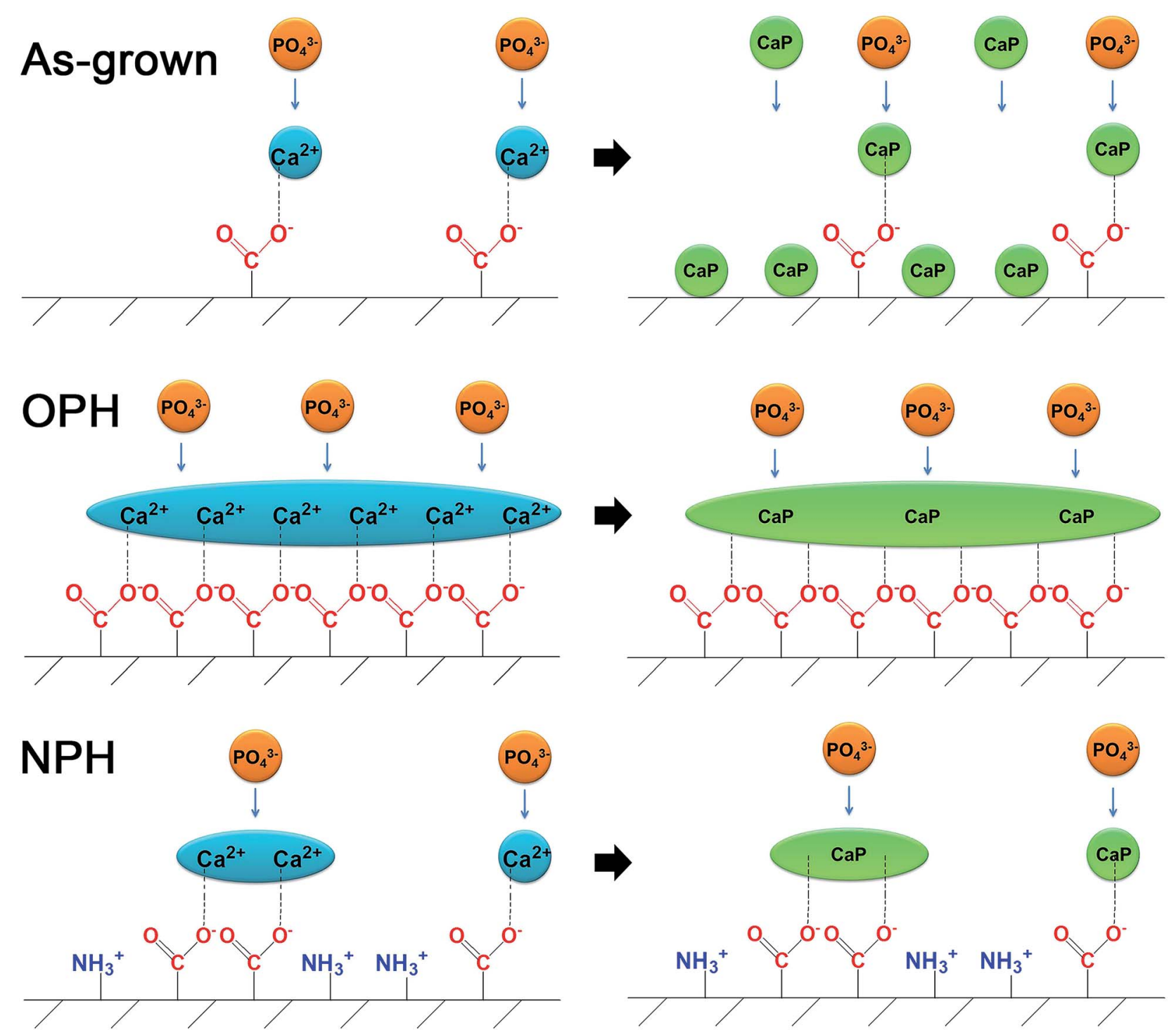

Fig. 6 The mechanism of mineralization on the surfaces of the three microsphere scaffolds.

carboxyl groups. ${ }^{35}$ Secondly, apatite was formed by nucleation in the supersaturation due to the high ionic strength, although As-grown had few nucleation sites. Apatite deposited on the surface and lacked electrostatic attraction. So the mineralization layer was easy to crack and peel off. Plasma treated surfaces had a high electrostatic attraction and strong bonding force on the contrary. Thus the apatite layer remained intact on OPH and $\mathrm{NPH}$. Therefore, plasma process was an appropriate technique to fabricate qualified apatite coatings. Moreover, since the scaffold was superficially porous, a novel porous apatite layer could be obtained.

\section{Conclusions}

In summary, normal and plasma treated superficially porous scaffolds were mineralized in the SBF. The results revealed that plasma treatment could little affect mechanical property and superficially pore structures of scaffolds, but promote the mineralization, especially for OPH. So As-grown scaffold just produced a smooth porous apatite layer, while OPH a rough porous one. In addition, plasma treatment enhanced the bonding force between apatite layer and substrate. All these difference in properties of the mineralization layer was probably because plasma treatment introduced polar domains onto the surface. Future work is to study the interaction between the resultant porous mineralization layer and the mesenchymal stem cell with respect to bone repair.

\section{Acknowledgements}

This work was financially supported by National Natural Science Foundation of China (No. 51372085, 21574045), the Guangzhou Science and Technology Program (No. 201508010060), the Shenzhen Science and Technology Program (No. JCYJ20150401145529010), Guangdong Natural Science Funds for Distinguished Young Scholar (No. 2016A030306018) 
and the Fundamental Research Funds for the Central Universities (No. 2013ZZ0005).

\section{Notes and references}

1 D. Cheng, X. Cao, H. Gao and Y. Wang, J. Mater. Chem. B, 2013, 1, 3322-3329.

2 B. A. Dalton, M. D. M. Evans, G. A. McFarland and J. G. Steele, J. Biomed. Mater. Res., 1999, 45, 384-394.

3 J. H. Fitton, B. A. Dalton, G. Beumer, G. Johnson, H. J. Griesser and J. G. Steele, J. Biomed. Mater. Res., 1998, 42, 245-257.

4 A. K. Salem, R. Stevens, R. G. Pearson, M. C. Davies, S. J. B. Tendler, C. J. Roberts, P. M. Williams and K. M. Shakesheff, J. Biomed. Mater. Res., 2002, 61, 212-217.

5 D. Cheng, X. Cao, H. Gao, J. Hou, W. Li, L. Hao and Y. Wang, RSC Adv., 2015, 5, 17466-17473.

6 Y. Kim and G. Kim, Colloids Surf., B, 2015, 125, 181-189.

7 L. Sun, C. C. Berndt, K. A. Gross and A. Kucuk, J. Biomed. Mater. Res., 2001, 58, 570-592.

8 P. K. Chu, J. Y. Chen, L. P. Wang and N. Huang, Mater. Sci. Eng., $R, 2002,36,143-206$.

9 T. Kokubo and H. Takadama, Biomaterials, 2006, 27, 29072915.

10 L. Kong, Y. Gao, G. Lu, Y. Gong, N. Zhao and X. Zhang, Eur. Polym. J., 2006, 42, 3171-3179.

11 Y. Guo, J. Lan, C. Zhang, M. Cao, Q. Cai and X. Yang, Appl. Surf. Sci., 2015, 349, 538-548.

12 Q. Liu, J. Ding, F. K. Mante, S. L. Wunder and G. R. Baran, Biomaterials, 2002, 23, 3103-3111.

13 J. Chen, B. Chu and B. S. Hsiao, J. Biomed. Mater. Res., Part A, 2006, 79, 307-317.

14 J. M. Goddard and J. H. Hotchkiss, Prog. Polym. Sci., 2007, 32, 698-725.

15 C.-M. Chan, T.-M. Ko and H. Hiraoka, Surf. Sci. Rep., 1996, 24, 1-54.

16 J. Yang, Y. Wan, J. Yang, J. Bei and S. Wang, J. Biomed. Mater. Res., Part A, 2003, 67, 139-1147.

17 X. Qu, W. Cui, F. Yang, C. Min, H. Shen, J. Bei and S. Wang, Biomaterials, 2007, 28, 9-18.

18 F. He, J. Li and J. Ye, Colloids Surf., B, 2013, 103, 209-216.

19 D. Cheng, X. Cao, H. Gao and Y. Wang, RSC Adv., 2013, 3, 6871-6878.

20 D. Cheng, J. Hou, L. Hao, X. Cao, H. Gao, X. Fu and Y. Wang, J. Biomed. Mater. Res., Part B, 2016, 104, 1056-1063.
21 F. Barrere, C. A. van Blitterswijk, K. de Groot and P. Layrolle, Biomaterials, 2002, 23, 1921-1930.

22 D. Cheng, H. Gao, L. Hao, X. Cao and Y. Wang, Mater. Lett., 2013, 111, 238-241.

23 H. Luo, G. Xiong, C. Zhang, D. Li, Y. Zhu, R. Guo and Y. Wan, Mater. Sci. Eng., C, 2015, 49, 526-533.

24 H. Koch, W. Kulisch, C. Popov, R. Merz, B. Merz and J. P. Reithmaier, Diamond Relat. Mater, , 2011, 20, 254-258.

25 J. F. Evans and T. Kuwana, Anal. Chem., 1979, 51, 358-365.

26 S. Delpeux, F. Beguin, R. Benoit, R. Erre, N. Manolova and I. Rashkov, Eur. Polym. J., 1998, 34, 905-915.

27 S. Torrengo, A. Miotello, L. Minati, I. Bernagozzi, M. Ferrari, M. Dipalo, E. Kohn and G. Speranza, Diamond Relat. Mater., 2011, 20, 990-994.

28 Y. Nitta, K. Okamoto, T. Nakatani, H. Hoshi, A. Homma, E. Tatsumi and Y. Taenaka, Diamond Relat. Mater., 2008, 17, 1972-1976.

29 G. Altankov, K. Richau and T. Groth, Materialwiss. Werkstofftech., 2003, 34, 1120-1128.

30 A. Voss, H. Wei, C. Müller, C. Popov, W. Kulisch, G. Ceccone, C. Ziegler, M. Stengl and J. P. Reithmaier, Diamond Relat. Mater., 2012, 26, 60-65.

31 B. C. Yang, J. Weng, X. D. Li and X. D. Zhang, J. Biomed. Mater. Res., 1999, 47, 213-219.

32 M. Tavafoghi and M. Cerruti, J. R. Soc., Interface, 2016, 13, 20160462-20160473.

33 M. Tavafoghi, N. Brodusch, R. Gauvin and M. Cerruti, J. $R$. Soc., Interface, 2016, 13, $20150986-20150997$.

34 M. Ndao, J. T. Ash, P. S. Stayton and G. P. Drobny, Surf. Sci., 2010, 604, L39-L42.

35 K. Sato, Y. Kumagai and J. Tanaka, J. Biomed. Mater. Res., 2000, 50, 16-20.

36 P. Zhu, Y. Masuda, T. Yonezawa and K. Koumoto, J. Am. Ceram. Soc., 2003, 86, 782-790.

37 D. Gebauer, M. Kellermeier, J. D. Gale, L. Bergström and H. Cölfen, Chem. Soc. Rev., 2014, 43, 2348.

38 D. Gebauer and H. Cölfen, Nano Today, 2011, 6, 564-584.

39 A. S. Posner and F. Betts, Acc. Chem. Res., 1975, 8, 273-281.

40 W. J. E. M. Habraken, J. Tao, L. J. Brylka, H. Friedrich, L. Bertinetti, A. S. Schenk, A. Verch, V. Dmitrovic, P. H. H. Bomans, P. M. Frederik, J. Laven, P. van der Schoot, B. Aichmayer, G. de With, J. J. DeYoreo and N. A. J. M. Soommerdijk, Nat. Commun., 2013, 4, 1507-1519. 41 Q. Cai, Q. Xu, Q. Feng, X. Cao, X. Yang and X. Deng, Appl. Surf. Sci., 2011, 257, 10109-10118. 